\title{
Opfergemeinschaften und Verschwörungsphantasmen. Reflexionen über pädagogische Praxis in der postnazistischen Gesellschaft
}

\author{
Enno Stünkel
}

\section{Turning a blind eye}

Der britische Psychoanalytiker John Steiner hat mit der Formulierung ,turning a blind eye" einen Mechanismus benannt, der es ermöglicht, ein anscheinend schon erkanntes Problem der Wahrnehmung zu entziehen. Das, was bewusst scheint, vielleicht sogar im Mittelpunkt der Aufmerksamkeit steht, verschwindet in dem Moment, da es angeschaut wird:

„I refer to this mechanism as turning a blind eye because I think this conveys the right degree of ambiguity as to how conscious or unconscious the knowledge is. At one extreme we are dealing with simple fraud where all the facts are not only accessible but have led to a conclusion which is then knowingly evaded. More often, however, we are vaguely aware that we chose not to look at facts without being conscious of what it is we are evading. These evasions may lead to a sense of dishonesty and to various manoeuvres which deny or conceal what has happened by creating a cover up."1

Manchmal bedarf es einer Gesellschaft, um ein Problem derart in den Blick zu nehmen, dass es darüber unsichtbar wird. Steiner zeigt das an Sophokles' König Ödipus. Dazu liest er das Drama nicht als Geschichte einer Aufklärung, sondern als Darstellung einer kollektiven Anstrengung, das, was offenkundig ist, nicht zu sehen. Ödipus ist hier nicht der Analytiker seiner Schuld, der, nach Wahrheit suchend, erkennt, dass er mit seiner Mutter geschlafen und seinen Vater getötet hat, vielmehr unternimmt Ödipus alles, um nicht zu wissen, was doch zu wissen wäre. Er verzichtet auf naheliegende Fragen und stellt Verbindungen nicht her. Eben dadurch vollzieht sich das Drama. Damit dieses Vermeiden von Wissen gelingen kann, müssen alle, müssen Iokaste und Kreon, muss der Chor sich auf entsprechende Weise verhalten. So entsteht in Steiners Aufsatz das Bild einer Gesellschaft, die gemeinsam und arbeitsteilig Einsicht vermeidet. Mit diesem Modell lässt sich beschreiben, wie die postnazistischen Gesellschaften ihren Antisemitismus vor sich selbst verborgen halten - den gegenwärtigen ebenso wie den geschichtlichen. Methodisch kann das Modell dazu anregen, für die Wahrnehmung und für die Analyse des Antisemitismus gerade auf die Abwehr- und Vermeidungs-

${ }_{1}^{1}$ John Steiner, Turning a blind Eye. The Cover Up for Oedipus, in: The International Review of Psycho Analysis 12 (1985), S. 161-172, hier S. 161. 
mechanismen $\mathrm{zu}$ achten und so besser $\mathrm{zu}$ erkennen, unter welchen Bedingungen Aufklärung nicht gelingt.

Zum Komplex des Antisemitismus gehören auch die Schwierigkeiten, ihn als solchen zu erkennen. Ein Bekenntnis ,gegen Antisemitismus“ geht nicht zwangsläufig mit einer Erkenntnis, was Antisemitismus geschichtlich und aktuell bedeutet, einher. Insbesondere die Vorstellungen ,jüdischer Macht“, die seit dem 19. Jahrhundert das Zentrum antisemitischer Projektion bestimmen und heute in Form von neuen Verschwörungsphantasmen und moralischen Erzählungen über das Böse in der Welt, in der Angst vor dem mächtigen Israel und der Verfolgung durch Juden wiederkehren, werden aber nicht als das erkannt was sie sind. Diese Vorstellungen sind in einem Maße irrational und gleichzeitig so sehr mit historischer und aktueller Gewalt verbunden, dass man den Eindruck gewinnen mag, dass dem gesellschaftlichen Wahngeschehen mit verdinglichendem und isolierendem Gestus begegnet wird, der bannen soll, was an Unheimlichem sonst unfassbar, tatsächlich aber unerträglich bekannt erschiene.

Diese Beobachtung möchte ich im Folgenden an zwei Begebenheiten entwickeln und auf ihre Auswirkungen auf eine mögliche pädagogische Praxis hin befragen, die sich bewusst sein muss, in einer Öffentlichkeit stattzufinden, in der auch die kritische Aufmerksamkeit auf Antisemitismus zu seiner Verstetigung beitragen kann. Es ist, als wirke die realitätszerstörende Macht des antisemitischen Ressentiments auch in den Bemühungen, diesem Ressentiment etwas entgegenzusetzen. Die großen, medialen Debatten - von der Paulskirchenrede Martin Walsers, über Günter Grass’ Gedicht „Was gesagt werden muß“ bis zu den Diskussionen um Jakob Augstein oder Xavier Naidoo - scheinen kaum Einsichten zu vermitteln. Das Modell des „,turning a blind eye“ mag eine Möglichkeit eröffnen, das nicht intendierte Zusammenwirken von Verleugnungen, Abspaltungen und Abwehrgesten so zu interpretieren, dass ein angemesseneres Verständnis der aktuellen Dynamik des Antisemitismus und der Gesellschaft, in der dieser wirkt, gelingt. Das sollte für eine pädagogische Praxis, die die antisemitische Tradition unterbrechen will, gerade in dem Moment wichtig sein, da sie sich als reflektierte Praxis zu verbreiten beginnt. ${ }^{2}$ Denn mit dem Zugewinn an historischem und sozialpsychologischen Wissen, das wir in den letzten Jahren erleben, geht eine Neuformierung des Widerstands gegen diese Einsichten einher - das zumindest legen die Erfahrungen aus der „Aufarbeitung“Geschichte der postnazistischen Gesellschaften nahe. Die verbreitete Unkenntnis von Wirkung und Funktion des Antisemitismus muss wohl noch immer (oder immer wieder) als Schutz vor gesellschaftlicher Selbsterkenntnis verstanden werden.

2 Vgl. dazu einführend und mit weiteren Hinweisen Meron Mendel/Astrid Messerschmidt (Hrsg.), Fragiler Konsens.

Antisemitismuskritische Bildung in der Migrationsgesellschaft, Frankfurt a. M. 2017. 


\section{Phantasien über Antisemiten - Wiederholungen}

Unter dem Titel „Heiliger Durchblick“ veröffentlichte die Cellesche Zeitung am 7. Juni 2014 eine Pfingstandacht des protestantischen Theologen Otmar Schulz. Der Text erschien unter der farbigen Reproduktion des Gemäldes „Pfingsten“ von Emil Nolde aus dem Jahr 1909. Damit bezog sich Schulz auf eine Debatte, die anlässlich der großen NoldeRetrospektive im Frankfurter Städel Museum zum wiederholten Mal sich an der Rolle Noldes im Nationalsozialismus entzündete. Der (postume) Erfolg des Malers Nolde beruhte nach 1945 darauf, dass der als im Nationalsozialismus verfemter Künstler galt; eine Legende, die ein Beispiel für die Leugnung und Umdeutung von nationalsozialistischer Geschichte ist. Den 2014 in Ausstellung und Katalog präsentierten Forschungsstand fasste die FAZ zusammen: „Nolde, in summa, war bis zum Kriegsende ein überzeugter Nationalsozialist und glühender Bewunderer Hitlers. Er war ein entschiedener Antisemit. Er hielt seine Verfemung durch die Kunstbürokratie für ein schreckliches Missverständnis, gegen das er beständig kämpfte. “3

Das ist der Hintergrund, vor dem die Pfingst-Andacht über das Nolde-Gemälde steht. In dem mit „Nachwort“ betitelten Teil, der fast die Hälfte des Textes umfasst, bezieht Schulz sich darauf: „Die Frage, ob Nolde Nazi war oder gar Antisemit, wird in jüngster Zeit wieder heftiger diskutiert. [...] Sie lässt sich auch in diesem Beitrag nicht umgehen. “4 Warum ist diese Frage nicht zu umgehen? Die Frage, ob „Nolde Nazi war“", bietet wenig Spielraum für die Diskussion. Seine Mitgliedschaft in der NSDAP bis 1945 ist dokumentiert. Auch die Zuspitzung, „Nazi oder gar Antisemit“ ist bemerkenswert, scheint sie doch zu implizieren, dass die Gruppe der Nazis größer war als die der Antisemiten. Der letzten zuzugehören, das insinuiert das ,gar", ist kaum denkbar. Ein Abwehrsignal ist gesetzt, das die folgenden Überlegungen leitet. Es geht Schulz um die Berechtigung des Begriffs „Antisemitismus“. Wir erfahren: „Noldes vermeintlicher „Antisemitismus' hatte eine Vorgeschichte." Schulz fasst sie so zusammen: Noldes „Gemälde Pfingsten wurde 1910 auf Anweisung von Max Liebermann aus der Ausstellung der Berliner Secession verbannt. Nolde lieferte sich anschließend einen heftigen Briefwechsel mit Liebermann, der ihn daraufhin aus der Secession ausschloss. Er hatte die Macht dazu, er war der Präsident.“ Es ist hier nicht der Ort, die kunsthistorische Geschichte des Konfliktes zwischen der Berliner Secession und den sich um 1910 entwickelnden Strömungen der (expressionistischen) Malerei nachzuzeichnen. Wichtig für unseren Zusammenhang ist, dass Schulz einen persönlichen und künstlerischen Konflikt zwischen Nolde und Liebermann als „Vorgeschichte“ eines schon in Anführungszeichen gesetzten Antisemitismus deutet, in diesem also Ausdruck

${ }^{3}$ Jochen Hieber, Der Erzähler Siegfried Lenz und sein Modell Emil Nolde, in: Frankfurter Allgemeine Zeitung, 26.4.2014, S. 9.

${ }^{4}$ Otmar Schulz, Heiliger Durchblick, in: Cellesche Zeitung, 7.6.2014, S. 1. So auch für die folgenden Zitate, wenn nicht anders angegeben. 
einer lebensgeschichtlichen Erfahrung sieht. Diese Lebensgeschichte wird von Schulz typisierend nachgezeichnet. Wir erfahren, dass Noldes Jugendjahre geprägt waren von „harter Arbeit und kargem Leben. Erst 1899 mit 32 Jahren, konnte er sich eine Malerschule leisten.“ Ganz anders sein Gegenspieler: „Liebermann, 20 Jahre älter als Nolde, stammte aus einem reichen Elternhaus, hatte alle Möglichkeiten, gute Schulen zu besuchen und sorglos zu studieren." Eine idealtypische Gegenüberstellung: Macht, Reichtum, Sorglosigkeit auf der einen, harte Arbeit und karges Leben auf der anderen Seite. Auch steht alt gegen jung. Schulz erklärt, was weiter geschah:

„Seinem Rauswurf aus der Berliner Secession wegen seines Pfingstbildes folgten weitere Auseinandersetzungen mit Max Liebermann und Paul Cassirer, dem führenden Kunsthändler, wie Liebermann ebenfalls aus reichem jüdischen Hause. Das waren die dominierenden Kräfte des damaligen Kunstmarktes, Liebermann als Maler, Cassirer als mächtiger Kunsthändler. Überdies war Liebermann eifriger Vertreter des französischen Impressionismus, den Nolde als ,undeutsch“verabscheute. Er suchte vielmehr nach einer eigenständigen ,deutschen' Ausdrucksform und meinte, sie im Expressionismus gefunden zu haben. Und eckte damit auch bei anderen (jüdischen) Kunsthändlern an. Es ist tragisch, dass diejenigen, die Nolde in seiner Karriere behinderten, Juden waren. Und das in einer Zeit, wo der Antisemitismus politisch gewollt war. Wir, die wir das Glück der späten Geburt haben, haben schnell den Stempel ,Antisemit‘ bei der Hand. Er muss nicht stimmen.“

Das ist die „Vorgeschichte“ des „,vermeintlichen ,Antisemitismus““. Aber der „Stempel ,Antisemit“ stimmt nicht. Warum nicht? Offenbar deswegen, weil das, was Nolde bestimmt, keine Ideologie war, sondern eine persönliche Erfahrung. Damit übernimmt Schulz selbst eine zentrale Rechtfertigungsfigur des Antisemitismus. Julijana Ranc hat deren Wirken, unter dem Begriff der „Kausaltäuschung“, so beschrieben:

„Wie alle Ressentimentgeleiteten [...] wollen Antisemiten deshalb auch das, was sie umtreibt, als etwas anderes verstehen oder verstanden wissen als das, was es ist: Nämlich als unvermittelte und kausal begründbare Reaktion auf ein Sosein oder Verhalten derjenigen, die sie in Wirklichkeit bereits durch das ,Giftauge des Ressentiments“ abschätzen und gegen die ihre Aversion - via Projektion - bereits disponiert ist. Die Genese ihrer Aversion (und der Aversion ihrer Brüder und Schwerstern im Geiste) gegenüber Juden machen sie kausal an diesen selbst fest und dichten ihnen dergestalt, implizit oder explizit, zugleich die Schuld am Antisemitismus an."

Warum aber greift Schulz zu einer solchen Argumentation, die darauf zielt, den „Stempel des Antisemitismus" als falsch zu erweisen und warum nimmt er in Kauf, dafür selbst noch einmal antisemitisch konnotierte Muster zu wiederholen? Es fällt auf, dass Schulz keine Argumente vorweist, die sich mit dem Begriff Antisemitismus auseinandersetzen und etwa einen Vergleich vornähmen zwischen dem, was eine antisemitische Position

${ }^{5}$ Julijana Ranc, „Eventuell nichtgewollter Antisemitismus“. Zur Kommunikation antisemitischer Ressentiments unter deutschen Durchschnittsbürgern, Münster 2016, S. $21 \mathrm{f}$. 
wäre und dem, was Nolde selbst vertritt. Nolde selbst kommt bei Schulz nicht zu Wort. Der Frage, worin Noldes Antisemitismus sich äußerte, wird ausgewichen, stattdessen wird dieser biografisch gerechtfertigt. Und als durch Erfahrung gerechtfertigt wird er abgetrennt von dem, was als ,politisch gewollter Antisemitismus“ firmiert. Behauptet wird, gegen die offensichtlichen Zeugnisse, dass Nolde mit diesem Antisemitismus nichts zu tun gehabt habe. Noch einmal, warum?

Würde Schulz mit seiner Argumentation durchdringen, dann wäre das Wissen, das mühsam und nach einer Geschichte des Ausblendens und des Umschreibens, der Lüge und der Auslassung verfügbar ist, wenigstens für den Fall Nolde, wieder zurückgenommen und der Stand der 1950er-Jahre wäre wiederhergestellt. Zu fragen ist aber, ob Argumentationen, die wie eine Wiederholung der Abwehrhaltungen der 1950erJahre anmuten, heute die gleiche Bedeutung haben wie damals. Jede Wiederholung verändert. Wenn es darum geht, die „überwertige Identifikation“ aufrechtzuerhalten, dann gilt diese jetzt der Gesellschaft, die für sich in Anspruch nimmt, Geschichte in einer vorbildlichen Weise „aufgearbeitet“ zu haben. Die Figur des Malers Emil Nolde selbst spielt eine Rolle in der Geschichte dieser Aufarbeitung. Sie ist, in der literarischen Umformung, die ihr Siegfried Lenz in dem 1968 erschienenen Roman „Deutschstunde“ gab, für Generationen in Westdeutschland Schullektüre gewesen. Im Roman wird aus dem immer durchscheinenden Vorbild Noldes der widerständige und vom Nationalsozialismus verfolgte Maler Max Ludwig Nansen. Lenz hat den Anspruch dieses Romans damit bezeichnet, Antworten zu finden auf die Frage, ,wie geschehen konnte, was in diesem Land geschehen ist" "6. Aber gerade in dem Anspruch, verstehen zu wollen, wie ,geschehen konnte, was in diesem Land geschehen ist", wird Wesentliches ausgeblendet. Fragen werden nicht gestellt, Umdeutungen akzeptiert, und das „was in diesem Land geschehen ist“ verändert seine Bedeutung ins Ungewisse. „Auf den sechshundert Seiten der ,Deutschstunde‘ fällt das Wort Jude nicht einziges Mal - und dies, obwohl die im bäuerlichen und kleinbürgerlichen Milieu angesiedelte Handlung sonst nichts und niemanden vergisst, nicht den Landpfarrer und den Dorfschullehrer, nicht den Parteibonzen und den Kriegsversehrten, nicht den Briefträger, den Kneipenwirt und den Heimatforscher [...] Norddeutsche Landjuden aber gibt es ebenso wenig wie völkischen Antisemitismus. "7 Wenn man das Bild der Schullektüre und das der geschichtlichen Realität übereinanderlegt, wird aus dem Antisemiten und Nationalsozialisten der in seiner Kreativität und seiner rebellischen Unangepasstheit vorbildliche Maler.

Schulz' späte Wiederholung der Schuldabwehrgeste muss verstanden werden als Versuch, diese Ausblendung und Umdeutung aufrechtzuerhalten. Sie aufzugeben würde etwas verändern. Die Frage, warum die „Aufarbeitung des Nationalsozialismus“ so wenig zu einem angemessenen Verständnis von Antisemitismus beigetragen hat, sollte hier

${ }^{6}$ Hieber, Der Erzähler.

${ }^{7}$ Ebd. 
nach Antworten suchen. Schulz formuliert in vorgeblicher Selbstanklage: „Wir, die wir das Glück der späten Geburt haben, haben schnell den Stempel ,Antisemit ' bei der Hand.“8 Das ,schnell bei der Hand“ formuliert ein Verbot, das die meisten, die in erster, zweiter oder dritter Generation nach der NS-Zeit in den postnazistischen Gesellschaften aufgewachsen sind, kennen. Es ist das intergenerationell ausgesprochene Verbot, über eine Zeit zu urteilen, die man nicht erlebt hat. Ein moralischer vorgetragener Anspruch darauf, nicht moralisch beurteilt zu werden, mit Folgen, die als Beschädigung des moralischen Urteilsvermögens weiterwirken. Moralische Fragen aber sind es, um die es Schulz in seiner Pfingstandacht geht.

Obwohl die Ausführungen zu Nolde als Nachwort abgesetzt sind, bilden sie einen integralen Teil der Andacht. Erst wenn man den ersten Teil hinzunimmt, wird deutlich, welche religiöse und moralische Bedeutung der Entgegensetzung von Nolde und Liebermann zukommt. Denn tatsächlich ist sie bereits in der Pfingstgeschichte, wie Schulz sie erzählt, vorweggenommen. Sie beginnt damit, dass die Jünger Jesu sich nach dessen Tod „hinter verschlossenen Türen“ verbergen, „während draußen ihre jüdischen Verwandten ,Schawuot ' feiern“. Der Geist kommt über die Jünger und sie verlassen ,ihr verschlossenes, enges Haus, treten hinaus in die Weite des Tempelberges und erzählen den Tausenden da draußen von Jesus, vom Kommen des Reiches, wo Frieden und Gerechtigkeit herrschen, wo Barmherzigkeit stärker ist als Gier, Zuversicht stärker als Hoffnungslosigkeit“.?

Wie ein Echo verhalten sich diese Entgegensetzungen zu dem, was wir über Noldes Leben erfahren haben. Draußen die heimlich feiernden Jünger, eingesperrt erst, dann heraustretend, die wie Nolde die „Frömmigkeit“ besitzen, die sich „aus Fantasie und Ekstase speist“. Damit ist eine Entgegensetzung etabliert, die, wie in alter Ersetzungstheologie, die Pole des Guten und Verworfenen auf Kirche und Synagoge, auf Christen und Juden verteilt. In einer Anwendung für heute wird diese Polarisierung im Text in Frageform aufgenommen:

„Wer hat zum Beispiel recht: Der Geist des Geldes, der Märkte, der Massentierhaltung, der Atomlobby? Oder der Geist des einfachen Lebens, der Bescheidenheit, des ehrenamtlichen Einsatzes? Oder hat das eine mit dem anderen nichts zu tun? Gilt nun der Geist der Konkurrenz und des Individualismus oder doch der einer gemeinsamen Suche nach gangbaren Wegen in eine gemeinsam gestaltete Zukunft?" 10

Die Fragen sind rhetorisch, der „Geist der Wahrheit“ und der „Geist des Lebens“ stehen gegen den „Geist des Geldes“. Über die Frage, so Schulz, wie man vom Geist des Geldes zum Geist der Wahrheit komme, „müssten wir gemeinsam nachdenken. Der Sache auf den Grund gehen - wie Nolde.“ Und genau das geschieht dann in dem „Nachwort: Nolde - ein Nazi?“ Hier erfolgt die Einsetzung. Mit dem Geist des Geldes sind „die

8 Schulz, Heiliger Durchblick.

${ }^{9}$ Ebd.

10 Ebd. 
dominierenden Kräfte des damaligen Kunstmarktes, Liebermann als Maler, Cassirer als mächtiger Kunsthändler" gemeint. Dagegen steht der Geist des einfachen Lebens: „Noldes Frömmigkeit, die sich aus Fantasie und Ekstase speiste, aus einer tiefen Innerlichkeit."11 Die Behauptung, Nolde sei kein Antisemit gewesen, dient offenbar dazu, den moralischen Impuls, den Schulz in Nolde sieht, aufrechterhalten zu können er soll als moralische Person gerettet werden.

Nolde selbst übrigens hat seine Auseinandersetzung mit Liebermann anders geschildert; er nannte sie „meine um 1910 geführten Kulturkämpfe gegen die herrschende Überfremdung in allem Künstlerischen und gegen die alles beherrschende jüdische Macht“. ${ }^{12}$ Dass Schulz das auslässt, scheint mir entscheidend. Was nicht in den Blick geraten soll, ist der Antisemitismus, der sich selbst als moralisch überlegen gegen die „allesbeherrschende jüdische Macht“ rechtfertigte.

Nichts davon steht für sich allein oder ist untypisch. Ähnliches z. B. geschieht in Silvio Viettas mit weitaus höherem intellektuellen Anspruch unternommenen Versuch zur Rettung von Martin Heideggers „Globalisierungskritik“, die auf die Diskussion um die postum veröffentlichten „Schwarzen Hefte“13 reagiert. Den dort offen zutage tretenden Antisemitismus deutet Vietta um in ,Judenkritik“. Auch dazu muss der paranoidideologische Kern des Antisemitismus - die Verschwörung des Weltjudentums ausgespart werden. So wird die Geschichte zu etwas, das beiseitegelassen werden kann, und wichtig wäre allein die Intention dessen, was Heidegger dachte oder meinte und nicht seine Zeitgenossenschaft zum Mord an den europäischen Juden. Es mag so aussehen, als wiederhole Vietta hier nur die Darstellung, die Heideggers Apologeten nach 1945 verbreiteten und nach der auf die kurzzeitige Nähe zum Nationalsozialismus eine innere und äußere Distanzierung folgte. Die Neufassung des Antisemitismus, nach der Shoah und im Wissen um sie, die Heidegger nach 1945 betrieben hat, wurde nicht benannt, ja vielleicht nicht einmal erkannt. Sie verfiel dem blinden Blick. Heidegger schrieb auf die Vorhaltungen Herbert Marcuses, dass ein Philosoph sich nicht täuschen könne „über ein Regime, das Millionen von Juden umgebracht hat - bloß weil sie Juden waren“ am 13. Mai 1948: „[...] ich kann nur hinzufügen, daß statt ,Juden“, Ostdeutsche“ zu stehen hat und das dann genauso gilt für einen der Alliierten" ". ${ }^{14}$

Diese Täter-Opfer-Umkehr ist nicht ungewöhnlich, Heidegger bei weitem nicht der Einzige, der darauf verfiel, aber offenbar ist das Wissen und das Bewusstsein davon, in welchem Ausmaß das geschah, nicht nur bei „Meisterdenkern“, sondern spontan überall im besiegten Deutschland, etwas, das immer noch abgewehrt wird. Was Donatella Di Cesare über Heidegger schreibt, charakterisiert eine verbreitete Haltung der unmittelbaren Nachkriegszeit: „Die Juden werden nazifiziert, während die Deutschen

11 Ebd.

12 Zit. n. Hieber, Erzähler.

13 Silvio Vietta, „Etwas rast um den Erdball...“ Martin Heidegger: Ambivalente Existenz und Globalisierungskritik, Paderborn 2015.

${ }^{14}$ Ebd., S. 80. 
gleichsam hebräisiert und zu Opfern gemacht werden - in einer Rollenverdrehung, die nach der Shoah auch in anderen Zusammenhängen erfolgreich wiederholt wurde." 15 Diese Umkehr wird bei Heidegger noch weiter aufgeladen und begründet. Seine „Zivilisationskritik“, von der Vietta spricht, setzt Heidegger fort. In den Bremer Vorträgen von 1949 erklärt er: „Ackerbau ist jetzt motorisierte Ernährungsindustrie, im Wesen das Selbe wie die Fabrikation von Leichen in Gaskammern und Vernichtungslagern, das Selbe wie die Blockade und Aushungerung von Ländern, das Selbe wie die Fabrikation von Wasserstoffbomben. "16 In dieser „Zivilisationskritik“ sind "Juden" Agenten der Moderne, oder in Heideggers Worten: „Die zeitweilige Machtsteigerung des Judentums hat aber darin ihren Grund, daß die Metaphysik des Abendlandes, zumal in ihrer neuzeitlichen Entfaltung, die Ansatzstelle bot für das Sichbreitmachen einer sonst leeren Rationalität und Rechenfähigkeit“. ${ }^{17}$ „Genau besehen“, schreibt Vietta dazu, ,ist dies - wie auch die frühere Juden-Kritik Heideggers - ein Stück Kulturkritik, kein biologischer Antisemitismus [...]." ${ }^{18}$ Der Zeitpunkt der Notiz über das „Sichbreitmachen“ fällt zusammen mit dem Beginn der Vernichtung des europäischen Judentums, etwas, worauf Vietta nicht hinweist, was er auch nicht zu bedenken scheint. Und er erwähnt nicht, welche Konsequenz diese „kulturkritische“ Identifizierung von Judentum und moderner „Rationalität und Rechenfähigkeit“ hat. Di Cesare fasst die Absicht dieser Argumentation, die Heidegger nach verlorenem Krieg fortspinnt, so zusammen: „Die Juden haben sich selbst vernichtet."19 In Heideggers eigenen Worten: „Wenn erst das wesenhaft ,Jüdische“ im metaphysischen Sinne gegen das Jüdische kämpft, ist der Höhepunkt der Selbstvernichtung in der Geschichte erreicht." 20

Das Anfang der 1950er-Jahre durchgeführte „Gruppenexperiment“ des Frankfurter Instituts für Sozialforschung bewahrte nicht nur entsprechendes Material, es bleibt ein methodisches Vorbild, wie auch heute an und mit den kurrenten Äußerungen zu arbeiten wäre, um Funktion und Wirkung des antisemitischen Syndroms in seiner gesellschaftlichen Dimension erkennen und bearbeiten zu können. ${ }^{21}$ Die Befassung mit dem historischen Material kann dann helfen, die Kontinuität des Antisemitismus zu verstehen, die darin besteht, die stereotypen Bilder über den Wandel der Zeiten konstant zu halten, sie aber aufzuladen mit der (verleugneten) Macht des Geschehenen. Die Juden

${ }^{15}$ Donatella Di Cesare, Heidegger, die Juden, die Shoah, Frankfurt a. M. 2016, S. 321.

16 Martin Heidegger, Bremer und Freiburger Vorträge (Gesamtausgabe 79), Frankfurt a. M. 2005, S. 27.

${ }_{17}$ Martin Heidegger, Überlegungen XII-XV. Schwarze Hefte 1939-1941 (Gesamtausgabe 96), Frankfurt a. M. 2014, S. 46, zit. n. Vietta, Etwas rast, S. 171.

18 Ebd.

${ }_{19}^{19}$ Di Cesare, Heidegger, S. 310.

${ }^{20}$ Martin Heidegger, Anmerkungen I-V. Schwarze Hefte 1942-1948 (Gesamtausgabe 97), Frankfurt a. M. 2015, S. 20, zit. n. ebd.

${ }_{21}$ Friedrich Pollock, Gruppenexperiment. Ein Studienbericht, Frankfurt a. M. 1955; Vgl. zu dem hier angerissenen Sachverhalt Jan Lohl, „Deutsche fordern: Juden raus“. Antisemitismus nach Auschwitz im Alltagsdiskurs der 1950er Jahre, in: Charlotte Busch/Martin Gehrlein/Tom David Uhlig (Hrsg.), Schiefheilungen. Zeitgenössische Betrachtungen über Antisemitismus, Wiesbaden 2016, S. 131-153. 
des antisemitischen Bedürfnisses sind so gleichzeitig Phantasien der Macht, als deren eigentliches Opfer der Antisemit sich imaginiert, und Erinnerung an die Schwäche derer, die umgebracht wurden. Diese widersinnige Vorstellung enthält die destruktive Macht des Antisemitismus, in der der Nationalsozialismus weiterwirkt. Wir müssen an aktuellen Äußerungen zu verstehen versuchen, was Saul Friedländer über den „Widerschein des Nationalsozialismus" formuliert hat:

„In erster Linie geht es mir um eine Form, der Realität sich zu stellen, die zugleich ein Ausweichen vor ihr ist, um die Neutralisierung der Vergangenheit, um die Verschleierung - ob nun bewusst oder nicht - dessen, was an dieser Vergangenheit unerträglich bleibt. Hauptsächlich geht es also um einen Vorgang in der Gegenwart. Doch so seltsam es auf den ersten Blick scheinen mag: Auch der Nazismus selbst verstand es, sich der Realität zu stellen und ihr gleichzeitig auszuweichen. "'22

Die Bereitschaft, das Verbrechen zu leugnen, die Opfer als Sieger und als Täter zu imaginieren, lässt sich als Wunsch nach Wiederholung verstehen. Deswegen auch ist die Ähnlichkeit zwischen Äußerungen der Nachkriegszeit und heutigen Argumentationen kein Hinweis auf geschichtliche Kontinuität, sondern als Aktualisierung zu verstehen. Der Wunsch besteht fort und im Versuch der Abwehr erneuert er sich - und damit auch die Schuld. Dunkel ist das dem antisemitischen Wahn bewusst: deutlich wird das in der Abwehr, mit dem die Kritik am Antisemitismus als Verfolgung wahrgenommen wird. Methodisch aber bedeutet das, dass für die Wahrnehmung und für die Analyse des gegenwärtigen Antisemitismus immer auf die Abwehr- und Vermeidungsmechanismen $\mathrm{zu}$ achten ist, um zu verstehen, was dem Blick entzogen wird. In Schulz’ „Heiliger Durchblick“" war es das Phantasma der , jüdischen Macht“.

\section{„Die sind doch selber schuld.“ - Opfervorstellungen}

Eine ähnliche Konstellation in einem völlig anderen Setting: Teilnehmende in einem von mir geleiteten Lehrgang des zweiten Bildungsweges sollten im Unterrichtsgespräch den Begriff „Satire“ klären. In diesem Zusammenhang fiel der Name der Zeitschrift Charlie Hebdo. Der Anschlag auf die Redaktion lag drei Monate zurück. Ein junger Mann, der im folgenden N. genannt wird, reagierte auf den Namen und rief: „Die sind doch selber schuld!“ Mit „die“" waren die Opfer des Attentats gemeint.

Ich hatte Gelegenheit, diese Unterrichtssituation in Workshops nachzustellen. Beobachtungen aus dieser Rekonstruktion fließen in meine Überlegungen zu dem ein, was dort geschah, was gesagt, was gemeint und was verstanden wurde. Auf mich wirkte dieses „Die sind selber schuld!“ wie ein Schlag. Die sofortige Folge der Äußerung (vielleicht auch eine Reaktion auf meine nicht geäußerte, aber vielleicht sichtbare oder spürbar Emotion) war Stille im Raum. Eine anhaltende Stille, in der sich ein Gespräch

22 Saul Friedländer, Kitsch und Tod. Der Widerschein des Nazismus, München 1986, S. 74f. 
entspann, an der sich außer N. und mir nur noch eine weitere Person beteiligte: ein Mann, der sich in eine solidarische Gemeinschaft mit N. begab. Zunächst fragte ich nach: „Wie meinen Sie das, selber schuld?“, worauf N. entgegnete: „Die wussten doch, was ihnen droht.“ Auf die anschließende Frage: „Wie hätten sie das wissen sollen?“ antwortete er: „Das ist wie an einem Becken mit Piranhas. Wer da den Fuß reinhält...“

Ich fühlte mich unsicher, ein Gefühl, das auch die Teilnehmenden der Workshops erlebten. Die Unsicherheit führt zu Fragen wie diese: Sollte man N. dazu bringen, dass er diese Äußerung zurücknimmt? Würde er dadurch zu einer Rechtfertigung seiner Position verleitet? Wollen wir noch verstehen, wollen wir urteilen oder ausschließen? Ich werde diese Fragen hier nicht beantworten, auch wenn sie berechtigt sind. Interessant ist, wie sich die Haltung zu N. veränderte, je nachdem, welche Informationen über ihn die Teilnehmenden erhielten (oder sich ausmalten). Die Frage aber, die ich in den Workshops stellte, lautete: Ist das eine antisemitische Äußerung?

Einig waren sich die Teilnehmenden über zweierlei: dass es sich um eine Rechtfertigung von Gewalt handelte und dass sie deswegen in einer Unterrichtssituation nicht unwidersprochen stehen bleiben dürfe. Ziemlich schnell wurde spekuliert, dass es sich um einen muslimischen Schüler handelt, der religiös sei. Wahrscheinlich teile er die Empörung oder die Wut über die Karikaturen, die den Propheten Mohammed verspotteten. - Aber Antisemitismus? Mancher fiel dann ein, dass der Anschlag, der sich am Tag nach den Morden in der Reaktion von Charlie Hebdo ereignete, auf einen koscheren Supermarkt zielte und dort Juden traf. Aber das war ja nicht das, was bei Charlie Hebdo geschah. Was macht einen Anschlag zu einem antisemitischen Anschlag? Und wie vermittelt sich das im Reden über den Anschlag?

Den Zusammenhang hatte - in der Unterrichtssituation - N. selbst hergestellt. Mein Ziel war zunächst, wenig reflektiert, eine Art Einigung darauf zu erzielen, dass es nicht richtig und auch nicht zu rechtfertigen sei, Menschen wegen ihrer Äußerungen umzubringen. N. wechselte die Argumentation. „Überall sonst werden Menschen umgebracht und das kümmert hier keinen. Muslime werden umgebracht, aber das ist egal. Und außerdem: Es ist doch klar, wer dahinter steckt: Das weiß doch jeder. Das waren die Juden."

Schilderte ich diesen Fortgang, oder spielte die Rekonstruktion die Situation bis zu diesem Punkt weiter, wurde der Bezug zum Antisemitismus offensichtlich. Aber musste dafür das Wort „die Juden“ tatsächlich fallen? Meinem Eindruck nach stand der Antisemitismus ähnlich offen im Raum wie die Bejahung der Gewalt. Denn als solches habe ich dieses „Die sind doch selber schuld“ verstanden. Nicht allein des Inhalts der Worte wegen, sondern wegen einer schwer zu beschreibenden emotionalen Tönung, mit der sie ausgesprochen wurden: aufgeregt und kalt. Danach überraschte mich die Aussage, „die Juden“" steckten dahinter, nicht mehr. Mit Methoden der Vorurteilsforschung aber wäre an der Aussage „Die sind doch selbst schuld“ Antisemitismus nicht zu erkennen. Meine Beobachtungen bei den Diskussionen, die ich über diese Szene führen konnte, verdichten sich zu folgender Vermutung: Der Versuch, mit einer Äußerung wie der geschilderten umzugehen, die eigene Irritation zu bewältigen und der Störung, die mit 
dieser Äußerung einhergeht pädagogisch zu begegnen, führt leicht zu Interpretationen, in denen Abspaltungen und Trennungen die Wahrnehmung des Antisemitismus verhindern. Der Fokus auf N., seine Intentionen und seine Biografie, verstellt den Blick auf das Wesentliche.

Genauer gesagt: Das geschah bereits in der ursprünglichen Unterrichtssituation. Da war es N. selbst, der versuchte, den mit seiner Äußerung „Das waren doch die Juden“ manifest gewordenen Antisemitismus wieder unsichtbar werden zu lassen. N. griff zu einer sprachlichen Korrektur: „Entschuldigung, nicht Juden. Ich habe Israel gemeint. Israel steckt dahinter.“ Ändert das etwas? Und hängt das von der Biografie, von seinen Erlebnissen ab? ,Jetzt stehe ich hier als der einzige Antisemit da“, sagte er etwas später. In der Rekonstruktion der Szene habe ich eine anrührende Geste erlebt. Eine Frau wandte sich demjenigen zu, der die Rolle des N. spielte, beugte sich zu ihm, machte sich klein und redete ihn mit einer Freundlichkeit an, in der ich Traurigkeit hörte und die mitfühlend gemeint war: „Du bist sicher auch mal Opfer gewesen“, sagte sie. Ich bin mir nicht sicher, ob das Mitgefühl oder die Trauer richtig zugeordnet war: Bewusst schien sie bezogen auf seine Vergangenheit. Die Deutung, von der sie ausging, lautete, dass seine Aggression, seine Lust an der Beschuldigung der Juden aus seinen Erfahrungen von Ausgrenzung und Diskriminierung rührte.

Auch scheint mir fraglich, ob die Geste und die damit verbundene Intervention helfen kann: denn das setzte voraus, dass N. das Hilfsangebot darin erkennen würde und nicht allein die Schwäche des sich Kleinmachens. Aber könnte es sein, dass die Trauer, unbewusst, nicht der Vergangenheit galt, sondern dem Ziel, das in der Äußerung lag: dass N. Teil einer Opfergemeinschaft werden würde, bereit, zu opfern und geopfert zu werden. Dazu kann einem ein Bild einfallen, wie das, das in Schulbüchern der palästinensischen Autonomiebehörde verbreitet wird. Es zeigt Jungen und Mädchen, gemeinsam in einer Klasse. Sie alle haben die Augen gerichtet auf den einzig leeren Platz, einen leeren, mit Blumen geschmückten Stuhl, ein Ort der Bewunderung und der Sehnsucht, bezeichnet mit dem Wort: Märtyrer. ${ }^{23}$

Die Trauer sollte, so denke ich, der Rechtfertigung des Mordes gelten und der Opferbereitschaft, die darin anklingt. Das heißt nicht, darin eine Drohung oder die Gefahr einer „Radikalisierung“ zu erkennen und den jungen Mann auf dem Weg in den Dschihad zu sehen. Auch das wäre eine Möglichkeit, sich durch übertriebene Angst, durch Hysterie und Zuschreibung der Wahrnehmung zu entziehen. Wahrzunehmen wäre: die Faszination für die Wiederholung von Gewalt und Opfer und gleichzeitig die Leugnung davon: es waren die Juden und es werden die Juden sein.

In der Äußerung von N., das war mein unmittelbarer und nachhaltiger Eindruck, ist Gewalt spürbar geworden. Aber zu unterscheiden ist zwischen der emotional verstandenen Bedeutung und der tatsächlichen Qualität in der äußeren Realität. Und da

${ }^{23}$ Eldad J. Pardo, Palestinian Elementary School Curriculum 2016-17. Radicalization and Revival of the PLO Program, Jerusalem 2017, S. 9. 
gilt: Die Äußerung selbst ist - in diesem Raum und in diesem Umfeld - keine Gewalt. „Die sind selber schuld“ und „das waren die Juden“ sind Äußerungen, die darauf hinweisen, dass in der Verschwörungsbehauptung die Freude über die Gewalt und die Leugnung der Täterschaft zusammengehen. Das lässt sich verallgemeinern: Verbalantisemitismen sind an die Gewalttat historisch gebunden. Diese Verbindung ist ain den psychischen Repräsentanzen bewahrt, auch wenn das Bewusstsein sie verneint. Das erklärt, warum diese Verbindung so leicht in den Fokus des blinden Blicks gerät. Beispielhaft ist das zu erkennen, wenn der Philosoph Georg Meggle in seinem den Antisemitismus definitorisch entwirklichenden Text „Wer ist Antisemit“ schreibt: „Zur Erinnerung: Ein Antisemit sein heißt, eine Einstellung zu haben, die zu Recht absolut moralisch verwerflich ist. Das direkte Spiegelbild davon ist: Einen anderen als ,Antisemiten` zu bezeichnen, ist einer der schlimmsten Vorwürfe, die man einem Mitmenschen gegenüber erheben kann. Vor dem Hintergrund von Auschwitz vielleicht der schlimmste überhaupt. Was man dabei fast immer vergisst, aber nie vergessen sollte: Auch Worte können töten. Rufmord - das ist keine bloße Metapher. TotschlagArgumente schlagen nicht nur Argumente tot. “24

In dieser Umkehr (Antisemiten haben eine Einstellung, wer jemanden einen Antisemiten nennt, tötet ihn) ist das Verhältnis zwischen Antisemitismus und Gewalt bewahrt, wird aber durch die Umkehr unkenntlich, das Opfer ist der, der (fälschlich?) als Antisemit bezeichnet wird. In anderen Verneinungen wird die Tat von der Intention getrennt oder übersehen, dass antisemitische Vorstellungen auch Folge, nicht allein Motiv von antisemitischen Taten sind. Die Annahme, dass Aufklärung über die Shoah allein geeignet sei, Antisemitismus zu verhindern, übersieht, dass Gewalt und Vernichtung Faszination und Ziel des Antisemitismus sind. Gerade deswegen könnte es wichtig sein, das cover up, das über der Verbindung von Antisemitismus und Gewalt liegt - und seit der Shoah über jener von Antisemitismus und Vernichtung - aufzuheben. Statt der verleugnenden Trennung zwischen Antisemitismus und Vernichtung, die typisch für die postnazistischen Gesellschaft ist, wäre es wichtig, die psychische Freiheit zu erlangen, in der zwischen Gedanke, Wort und Tat zu unterscheiden ist und die es ermöglicht, die magische Verbindung zwischen Gedanke und Tat zu lösen, die auch dem Verschwörungsglauben zugrunde liegt und stattdessen die Realgeschichte der Gewalt als Grund der eigenen Phantasien zu erkennen. Das wäre eine Möglichkeit, antisemitische Ressentiments aufzugeben. Forderungen aber, Äußerungen zu strafen, kommen dem autoritären Wunsch entgegen, der vom Antisemitismus nicht zu trennen ist.

In der (ursprünglichen und reinszenierten) Unterrichtssituation blieb die Unsicherheit, wie angemessen zu reagieren wäre. Heike Radvan hat Fragen, die sich in diesem Zusammenhang stellen, zusammengestellt:

24 Georg Meggle, Wer ist Antisemit?, 2008, S. 14f., [https://www.sozphil.uni-leipzig.de/cm/philosophie/ files/2013/01/Wer-ist-Antisemit-08.pdf], eingesehen 13.7.2020. 
„Wie reagiere ich als Pädagogin auf eine antisemitische Aussage? Ist es sinnvoll, deren Ursprung und Motivation zu ergründen? Verbiete ich solche Äußerungen generell oder ist es zielführender, auf deren ,falsche Inhalte ${ }^{6} \mathrm{zu}$ verweisen? Ist es eine Option, das Statement zu ,überhören', da es sich um eine ,schlichte Provokation' gehandelt haben kann?" 25

Darin liegt der verständliche Wunsch nach einer Handlungsanweisung oder einem Rezept: Was wäre die richtige Verhaltensweise? Die Gefahr eines solchen Rezeptes liegt darin, anzunehmen, wir - nicht nur die pädagogisch Handelnden in der postnazistischen Gesellschaft - hätten im Wesentlichen schon verstanden, was Antisemitismus heute meint.

Die Metapher vom „blind eye“ lenkt aber demgegenüber die Aufmerksamkeit gerade auf die Möglichkeiten, die Bedeutung einer Äußerung tatsächlich zu überhören und den Antisemitismus damit zugleich zu übersehen. Naheliegend ist es, die Selbstrechtfertigung des N. aufzugreifen. Ändert sich nicht der Gehalt seiner Aussage tatsächlich, wenn nicht von Juden, sondern von Israel die Rede ist?

Macht die Formulierung nicht den Unterschied zwischen Kritik und Antisemitismus aus? Die scheinbar so schwierige Trennung von „Kritik an Israel“ und Antisemitismus bestimmt die gesellschaftliche Debatte über Antisemitismus in einer Vehemenz, dass die Frage danach zu einem bestimmenden Motiv der Abwehr geworden ist. Im hier geschilderten Fall verändert das Ersetzen des einen Worts durch das andere nichts am Gehalt der Aussage. Der Gehalt ist fixiert in der Phantasie, in der ein islamistischer Anschlag verschwindet und an seine Stelle eine Verschwörung tritt. Das aber zu verstehen fällt immer dann schwer, wenn Antisemitismus rationalisierend und verengend verstanden wird als die (mehr oder minder) hasserfüllte Ablehnung von realen Jüdinnen und Juden, die auf Vorurteilen oder auf falsch verallgemeinerter Erfahrung beruhe - und eben nicht als eine falsche und auf Gewalt zielende Scheinerklärung von Gesellschaft durch das Wirken geheimer und zu enttarnender Kräfte. Ob in antisemitischer Rede „Juden“ oder „Zionisten“ benannt werden, macht keinen Unterschied. Es scheint aber genau diese Phantasie von der Verschwörung zu sein, die Irritationen auslöst und (nicht nur) Pädagoginnen und Pädagogen überfordert.

Juljana Ranc hat in ihrer wichtigen Studie darauf hingewiesen, dass in den Gruppengesprächen, die sie auf antijüdische Ressentiments untersucht hat, „[o]ffenkundig verschwörungstheoretische Themensetzungen über Juden [...] gruppenübergreifend nur wenig resonanz- und interaktiv nicht etablierungsfähig" 26 waren. Dies deckt sich auch mit meiner Beobachtung bei Workshops mit Pädagoginnen und Pädagogen, bei denen zunächst kaum Wissen um „Verschwörungstheorien“

\footnotetext{
${ }^{25}$ Heike Radvan, Die Bedeutung von Kommunikation im Umgang mit Antisemitismus am Beispiel der offenen Jugendarbeit, in: Maron Mendel/Astrid Messerschmidt (Hrsg.), Fragiler Konsens. Antisemitismuskritische Bildung in der Migrationsgesellschaft, Frankfurt a. M. 2017, S. 43.

${ }^{26}$ Ranc, „Eventuell nichtgewollter Antisemitismus"rhebun, S. 48.
} 
vorhanden schien. Doch diese Beobachtung täuscht und es scheint sich eher um ein tabuisiertes Wissen zu handeln. Auch Ranc beobachtete hellhörig weiter:

„Allerdings schienen die meisten weitaus mehr über die Existenz und den Inhalt antijüdischer Verschwörungstheorien zu wissen als sie zuzugeben bereit waren. Dafür spricht eine oft schon im Ansatz, nach den ersten vagen Andeutungen greifbare atmosphärische Anspannung, eine vorausahnende Wachsamkeit in den Gesichtern so mancher Mitdiskutanten, sprechen kommunikative Verhaltenheit sowie sichtlich peinlich berührtes Schweigen. Auch kam es vor, daß man denjenigen, die sich gar zu weit aus dem Fenster zu lehnen drohten, eilfertig zur Seite sprang, etwa durch raschen Themenwechsel. Oder man ruderte gemeinsam ein Stück zurück. Wer ihrer Verpöntheit dergestalt Rechnung trägt, der kennt antijüdische Verschwörungstheorien. "27

Das muss, wie auch Ranc betont, nicht als Einverständnis gewertet werden. Ich schlage aber vor, es als Wunsch zu interpretieren, davon nichts wissen zu müssen, eben als ein Mechanismus des blinden Blicks. ${ }^{28}$ Das Wissen um die Virulenz antisemitischer Verschwörungsideologien, um ihre Quellen und jene, die sie propagieren, muss vorhanden sein, um in angemessener Weise einer Äußerung wie der von N. zu begegnen. Sie kommt weder aus dem Nichts noch ist sie das originelle Produkt der Phantasie dieses individuellen jungen Mannes. Wer nicht weiß, oder nicht wissen will, in welchen Zusammenhängen diese Verschwörungsbehauptungen kursieren, wird sie in ihrer gesellschaftlichen Bedeutung und Dynamik nicht verstehen. Trennungen und Zuweisungen liegen dann nahe, ähnlich der, die Otmar Schulz vornahm zwischen dem „politisch gewollten Antisemitismus“ und dem, was Nolde antrieb. Im Falle von N. und seiner Äußerung wäre es falsch, von einem importierten Antisemitismus zu sprechen, und zwar obwohl die Behauptung, Israel stecke hinter dem Anschlag, in Medien des Nahen Ostens prominent verbreitet wird.

Tatsächlich sieht das, was N. sagte, als Karikatur dargestellt so aus: Vor dem Hintergrund einer Stadtsilhouette, durch den Eiffelturm als Paris kenntlich, gehen zwei schwarz gekleidete und vermummte Männer, die gerade ein Auto verlassen haben, mit ihren Gewehren im Anschlag auf ein Tor zu, über dem der Schriftzug „Charlie Hebdo“ den Ort des Anschlags bezeichnet. An den Männern und an dem Auto sind Fäden befestigt, die zu einem einfachen hölzernen Spielkreuz führen und das von der Hand eines Marionettenspielers gehalten wird. Der Spieler selbst ist nicht zu sehen, der Arm ragt aus dem Himmel in den Bildraum und ist am Revers mit dem Davidstern kenntlich gemacht. Eine derartige Karikatur wurde über die iranische Seite irib.ir verbreitet.

${ }^{27}$ Ebd., S. 49, Hervorh. im Original.

28 Die Gespräche wurden 2005 bis 2007 geführt. Es ist zu vermuten, dass gesellschaftliche Veränderungen und die mediale Verfügbarkeit von Verschwörungsideologien das Sprechen darüber in den letzten Jahren verändert haben. Das ist ein Hinweis darauf, wie wichtig es für die kritische Auseinandersetzung mit Antisemitismus ist, seismisch genau Veränderungen zu registrieren und den Antisemitismus gerade nicht für etwas Unveränderliches zu nehmen - entgegen allem Augenschein. 
Die Behauptung, bei dem Anschlag auf Charlie Hebdo handele es sich nicht um das, was uns glauben gemacht wird, findet sich auch auf den Seiten eines im Sommer 2017 gegründeten Internetmagazins, das sich selbst als „Magazin für die kritische Masse“ bezeichnet:

„Bei Charlie Hebdo öffnen sich zufällig drei Mal sonst verschlossene Türen, und dieses Wunder muss geplant gewesen sein. Andernfalls, bei Türsprengungen, Geballer, Geiselnahmen und Geschrei, wären ja der Wachmann alarmiert worden, die Redaktionsmitglieder auf das Dach geflüchtet, die allgegenwärtige Polizei im Herzen der Hauptstadt Paris alarmiert worden. Kinder würden sagen: also ein Märchen..."29 Eine Andeutung genügt - wer das „Wunder“ geplant hat, wird nicht benannt. In welchem Verhältnis steht sie zu der manifest antisemitischen Verschwörungstheorie? Wahrscheinlich würde sich der Verfasser dagegen verwahren, wollte man ihm die antisemitische Intention unterstellen, die der anonyme Zeichner in der Karikatur offenbart. Mit der vorgeblichen Naivität eines Kinderblicks weist der Autor nur auf offenkundige Ungereimtheiten hin, die schon ein Kind erkennen ließen, dass hier etwas nicht stimme. Die Leichtigkeit, mit der die Lüge aufgedeckt wird, der große Plan durchschaut wird, erreicht der Autor durch die Perspektive eines Kindes. Dahinter steht eine Infantilisierung, die durch ihr Ausstellen überspielt wird. Denn die Bereitschaft, an Verschwörungsbehauptungen dieser Art zu glauben setzt tatsächlich eine Regression voraus: rationale Techniken verlieren an Bedeutung. Sprachliche und argumentative Mängel, die offenkundig sind, werden nicht mehr wahrgenommen und die Fähigkeit zur Realitätsprüfung lässt nach. Dem mit Medienkritik und Aufklärung entgegenzuwirken, stößt schnell an Grenzen. Wer Verschwörungsideologien anhängt, spielt mit oder verfällt magischem Denken. Aggression gegen die, die einen manipulieren, wird zur Selbstverteidigung. Der autoritäre Glaube an beliebige Experten geht einher mit einer Absage an Rationalität und Wissenschaft und verrät, dass der rationale, planende, verändernde Eingriff in die Welt für unmöglich oder für ethisch verboten gehalten wird. So sehr anzunehmen ist, dass all das Resultat einer Gesellschaft ist, in der Eigenwirksamkeit, Selbstbestimmtheit und Handlungsfreiheit real nicht erfahren werden, kritisiert eben Verschwörungsideologie jene Unfreiheit nicht, sondern behauptet eine Welt, in der der Ausweg in der Demaskierung der manipulierenden und herrschenden Kräfte liegt: dieser Ausweg aber ist selbst nichts anderes als magisches Denken. Enthüllen und das Brechen der Macht fallen zusammen, aber nur in der Phantasie. Daher ist der Verschwörungsglaube stete Quelle von Frustration - oder Erinnerung an und Versprechen von Gewalt. Der Aufstand, der geträumt oder geführt wird, richtet sich real gegen Schutzlose. Das ist die historische Wahrheit des Antisemitismus, um die alle wissen. Er richtet sich auch gegen die psychischen Repräsentanzen von Macht, die gefürchtet werden (als Manipulation, als Versagung, als Strafe), die aber als Rationalität,

29 Andreas Hauß, Terror-Logik, in: rubikon.news, 7.6.2017, [https://www.rubikon.news/artikel/terroralarm], eingesehen 13.7.2020. 
als Gesetz und als Empathie tendenziell fähig wären, als Bündnispartner für Aufklärung und $\mathrm{zu}$ erreichende Freiheit $\mathrm{zu}$ wirken. Das beschädigt auch die Fähigkeit zum moralischen Urteilen.

\section{Die moralische Geste}

Die Vorstellung einer umfassenden, die Geschichte bestimmenden, die Welt bedrohenden Macht steht im Zentrum des modernen Antisemitismus. Für den postnazistischen Antisemitismus bedeutet das, dass die Leugnung der Shoah die originäre Verschwörungsphantasie nach 1945 wird: die Phantasie des triumphierenden, des mächtigen Juden entsteht in ihr gegen alle Tatsächlichkeit neu. Und daher ist das Motiv der Schuldumkehr so zentral: es entlastet nicht nur die Täter und die, die mit ihnen familiär, generationell, gesellschaftlich und moralisch verbunden sind. Es stellt auch den wahnhaften Charakter des Antisemitismus (wieder) her. Worin besteht die Macht - nicht die Macht der imaginierten Juden, sondern die Macht dieser Vorstellung? Was erhält sie, ihrer Unsinnigkeit zum Trotz, am Leben? Und wie geht man heute mit dieser Vorstellung um? Ich habe mit zwei Beispielen zu zeigen versucht, dass das „blind eye“ angewiesen ist auf genau die Ausblendung dessen, was den modernen Antisemitismus ausmacht: die Vorstellung von der geheimen, erst aufzudeckenden jüdischen Macht. Dieses Tabu schützt den Antisemitismus. Es blendet aus, was die antisemitische Vernichtungswut im Nationalsozialismus antrieb, jene pervertierte Moral, die in den Juden die Feinde schlechthin ausmachte, die Feinde nicht allein der Deutschen, sondern aller Völker, als Kriegstreiber, als die Macht hinter dem Finanzkapital, den Plutokratien und dem Bolschewismus. Ausgeblendet wird so die Selbstwahrnehmung der Antisemiten: für eine Erlösung der Welt zu kämpfen, sich gegen die Mächtigen aufzulehnen. Unverstanden bleibt das Rebellische des antisemitischen Affekts. Was bleibt, ist ein Zerrbild des Antisemitismus. Und leicht wird aus der gesellschaftlichen Ablehnung des Antisemitismus, wie sie seit 1945 gilt, die Ablehnung allein dieses Zerrbildes von Antisemitismus, das so erfolgreich ist, weil es den historischen Antisemitismus vom heutigen trennt, und den historischen um seinen Kern bringt. Dieses Zerrbild ist gleichermaßen falsch für die NS-Zeit wie für die heutigen, postnationalsozialistischen Zeiten. Es entzieht die andauernde Verschmelzung von Ideologie und Gewalt dem Blick. Der übermächtige Feind, den es zu enttarnen gilt, war real schutzlos. Dieses Wissen ist - maskiert aber verfügbar - in die heutigen antisemitischen Verschwörungsideologien eingegangen. Das könnte erklären, warum der offenkundig unsinnige Gestus, der in der behaupteten kinderleichten Enttarnung der doch überaus Mächtigen besteht, so attraktiv ist. Ein NS-Propagandaplakat von 1943 zeigt, wie ein Arm einen Vorhang beiseite zieht, der das Gesicht eines als „Kriegsanstifter“ und „Kriegsverlängerer“ bezeichneten Juden 
entblößt und dem entschlossen die Fäuste drohen. ${ }^{30}$ Diese Geste des Enttarnens ist das, was Verschwörungsideologien ausmacht. Man muss das Zusammendenken mit dem Morden der Wehrlosen, das gleichzeitig stattfand. Es ist diese Verbindung, die in unserer Wahrnehmung tabuisiert wird. Mit den Bildern der Opfer, der Überlebenden verbinden wir das Wissen um die absolute Hilflosigkeit. Der Wahn, in ihnen die Herren der Welt zu sehen, bedarf der Trennung und der neuen Codierung. Die Geschichte der Verwendung des Begriffs „Zionismus“ in der Nachkriegszeit ist ein beredtes Beispiel für eine solche Neu-Codierung. Noch im Angesicht der Bilder aus den Vernichtungslagern beginnt eine „neue“ Geschichte der Umkehrungen, die bis heute fortgeschrieben wird. Ein aktuelles Beispiel für eine solche Neu-Codierung - das als gesprochenes Dokument mehr an Einsicht vermitteln kann, als die folgende Transkription - stammt aus der Tonne des verschwörungstheoretischen „Mülltrenners“31 KenFM. Der emotionale Gehalt, im Text zu erkennen, wird von der Stimme übertragen, die in einem knapp einstündigen Stakkato Auskunft über das Verhältnis von Hass und Kälte sowie von Phantasie und Moral gibt:

„Zionismus ist im Kern eine Ideologie, die der der Herrenrasse verdammt ähnlich ist. Beide Ideologien gehen und gingen davon aus, man selber würde den Übermenschen darstellen, der das von Gott gegebene Recht besäße, sich über andere, also Restmenschen zu erheben, um diese zu beherrschen. Das auserwählte Volk ist die andere Seite einer Medaille, der in Nazi-Deutschland die Aufschrift ,Herrenrasse‘ eingraviert war. Heil Hitler und Heiliges Land sind primitive Blut-und-Boden-Betriebssysteme. "32

Ken Jebsen, Autor und Sprecher dieses Textes, geriert sich in den letzten Jahren regelmäßig als Opfer unbegründeter oder falscher Antisemitismusvorwürfe. Diejenigen, die ihn verteidigen, vermeiden es, sich mit Aussagen wie den zitierten auseinanderzusetzen. Jebsens Internetprojekt KenFM ist gleichzeitig eines der Zentren, um die sich der vielgestaltige Verweisstruktur der Verschwörungsszenen dreht. Wenn auch die Intentionen der Schuldabwehr, die Täter-Opfer-Umkehr unübersehbar den Text strukturieren, sollte der aggressive Charakter, der auf die Gegenwart zielt, nicht übersehen werden. Ken Jebsen betreibt nicht vorrangig Geschichtspolitik, auch dann nicht, wenn er die USA und die Federal Reserve Bank als Drahtzieher hinter den Weltkriegen darstellt. ${ }^{33} \mathrm{Zu}$ erkennen ist hier vielmehr die Ähnlichkeit, in der die Umkehr, auch wenn sie unterschiedlichen Intentionen folgt, in der antisemitischen Aufladung übereinstimmt. Nicht zufällig steht der Massenmord, den Islamisten (und damit Anhänger einer Ideologie, in der Antisemitismus zentral ist) am 11. September 2001 in

\footnotetext{
${ }^{30}$ Hans Schweitzer [Mjölnir], Der Jude: Kriegsanstifter, Kriegsverlängerer, ca. 1943, [https://www.warmuseum.ca/collections/artifact/1023686], eingesehen 13.7.2020.

${ }^{31}$ Kurze Beschreibung über das Portal KenFM.de, in: KenFM.de, o. D., [https://kenfm.de], eingesehen 13.7.2020.

32 Die deutsch-israelische Freundschaft, in: KenFM.de, 20.11.2012, [https://kenfm.de/deutsch-israelische], eingesehen 13.7.2020.

33 Siehe exemplarisch: Schein-Demokratie, in: KenFM.de, 31.4.2014, [https://kenfm.de/kenfm-ueber-scheindemokratie], eingesehen 13.7.2020.
} 
den USA begingen, am Beginn einer neuen Virulenz von Verschwörungsideologien, mit denen die Täterschaft (oder gleich die Tat) in Abrede gestellt wird. Auch für KenFM ist das ein zentrales Thema - und es bedarf einer blindäugigen Trennung der Themen, um nicht den Antisemitismus als verbindendes Motiv zu erkennen. ${ }^{34}$

In der postnationalsozialistischen Ablehnung des Antisemitismus wird die gesellschaftliche Einigkeit erkauft durch das gemeinsame Nicht-Hinsehen auf diesen Zusammenhang. So bleibt unbegriffen, dass eben der Hass auf Israel, die Leugnung der Täterschaft von Islamisten und die Mobilisierung gegen die „Globalisten“" zusammengehören - und eine klar erkennbare gemeinsame inhaltliche Bestimmung aufweisen. In dieser Neuerfindung antisemitischer Topoi für unsere Zeit sind nicht die Motive neu - neu aber ist ihre gesellschaftliche, geschichtliche und emotionale Aufladung. Sie zu verstehen, kann nicht Aufgabe der Pädagogik allein sein. Ihre Rolle wäre es aber, Reflexionsräume zu eröffnen, in der die Wahrnehmung blinder Flecken möglich wird. Dazu muss aber die Abwehr, die sich in der Leugnung der Zusammenhänge ausdrückt, gezielt in den Blick genommen werden.

\section{Schluss}

Die moralische Rettung der eigenen Haltung - das sollten die Beispiele zeigen - verkennt den Antisemitismus, um ihn nicht in den eigenen Motiven erkennen zu müssen. So wird ein Text wie jener von Jebsen, genauso wie das pfingstliche Plädoyer gegen den „Geist des Geldes, der Märkte, der Massentierhaltung, der Atomlobby“ und für den „Geist des einfachen Lebens, der Bescheidenheit“ nicht einfach durch die vorgebrachten Antisemitismen kontaminiert - sondern sie erscheinen als moralisch richtig und als Ausdruck kritischen Denkens. Dadurch aber, dass in diesem Sprechen antisemitisches Denken neu aufgeladen und geprägt wird, geht Gegenwärtiges in die Stereotype ein und wird von denen, die ihm anhängen, wider die geschichtliche Erfahrung rationalisiert und verteidigt. Heute sehen wir, gerade in den Debatten um den Antisemitismus, dass unter der Forderung, man müsse doch sagen dürfen, moralische Ansprüche formuliert werden. „Beunruhigend an der neuen Debatte ist jedoch,“ schrieb Werner Konitzer bereits 2005, „dass diese Operation scheinbar unlösbar verknüpft ist mit der Frage danach, ob man überhaupt, und wenn ja, was man gegen Israel, gegen das Judentum oder gegen Juden sagen darf. Diese Verknüpfung geht, so vermute ich, auf die enge Verschränkung von Moral und Verfolgung zurück, die der Nationalsozialismus nicht erst geschaffen, sondern nur radikalisiert hat. Auf sie reagierte nach dem Ende des Zweiten Weltkriegs jenes ,kommunikative Beschweigen' [...], in dem sich trotz gleichzeitiger ideologischer

${ }^{34}$ Für das Verhältnis von Verschwörungsideologie und (verdrängter) Gewalt ist der Effekt lehrreich, den Daniele Ganser bei seinen Vorträgen erzielt. Das Bild der Trümmerstätte im Stadtzentrum New Yorks löst bei den Zuhörerinnen und Zuhörern Lachen aus. Daniele Ganser, Wir wurden alle getäuscht, in: YouTube, 25.4.2017, [https://youtu.be/klYEWL-81GE], eingesehen 13.7.2020. 
Distanzierung unschwer eine Modifizierung der alten Komplizengemeinschaft unter den Gegebenheiten eines reformierten Moralsystems erkennen lässt. In den letzten Jahren wurde diese Symptomatik zusehends durch eine Konzeption ersetzt, die Auschwitz zu einer Art von Gründungsmythos der Bundesrepublik Deutschland adelt. An die Auseinandersetzung mit der alten, tradierten Moral trat etwas, das man als das ,Tabu des Antisemitismus' bis heute zu bezeichnen nicht aufgehört hat. " 35

Dieses Tabu, das aus der ausgebliebenen Auseinandersetzung folgt, könnte sich heute als der blinde Fleck entpuppen, der die Errungenschaften auch der kritischen pädagogischen Intervention gegen den Antisemitismus verunmöglicht. Der sich quer zu politische Zuordnungen und über unterschiedliche Intentionen hinweg herstellende Zusammenhang des antisemitischen Syndroms tritt als moralische Befreiung auf. Die gesellschaftlichen und psychologischen Bedingungen, die es erlauben, Befreiung als Opfer zu ersehnen, bilden immer wieder einen erneuerten Anreiz, dessen Dynamik nicht absehbar ist, sich in die antisemitische Tradition einzureihen. Sie erlaubt es, die eigenen Vernichtungswünsche als moralische Geste zu erleben. Der wahnhafte Blick auf jüdische Israelis, wie er in Jebsens Text aufscheint, ist unschwer als Beschwörung von Gewalt gegen das „,auserwählte Volk“ zu dechiffrieren. Nichts daran ist, was zu sein die Rede vorgibt: Kritik. Es handelt sich um eine Phantasie, in der das Böse enttarnt wird - die Macht, die uns alle im Griff hält und die uns manipuliert, findet ihren Widerpart in einem Wir, das sich als Opfer wähnt.

Auch wenn das Wissen um die historische Realität der Vernichtung aus dem Bewusstsein verdrängt und abgewehrt wird, so kann der antisemitische Wahn im Postnazismus dennoch nicht mehr hinter diese historische Erfahrung zurück. Kritik, die es ermöglicht, der antisemitischen Tradition $\mathrm{zu}$ entgehen, muss diesen Zusammenhang von Irrationalität und Gewalt benennen, um ihn zu unterbrechen. Dazu bedarf es der gesellschaftlichen Reflexion, für die, kontrastierend zu den Fortschritten, die es gerade in der Pädagogik gegen Antisemitismus in jüngster Zeit gibt, die Gelegenheiten in der Gesellschaft spärlicher werden. Immerhin gibt es mittlerweile methodisch kluge, reflektierte Modelle eines kritischen Umgangs mit Antisemitismus in Bildungseinrichtungen. In verschiedenen gesellschaftlichen Milieus gelingt es, Haltungen zu entwickeln, die antisemitische Reflexe und Traditionen unterbrechen. Dagegen aber entwickeln sich wieder Abwehrbewegungen - weswegen die scheinbare Wiederkehr des Überholten und Obsoleten eben keine Wiederholungen sind, sondern jeweilige Aktualisierungen aus gegenwärtiger schlechter Vergesellschaftung. Der Begriff des Antisemitismus bezeichnet das Wirken eines Wiederholungszwangs, dessen Dynamik zu verkennen eine weitere Möglichkeit ist, dem Irrationalen auszuweichen, anstatt sich ihm zu widersetzen.

35 Werner Konitzer, Antisemitismus und Moral. Einige Überlegungen, in: Mittelweg 3614 (2005), Heft 2, S. 24-35, hier S. 35 . 
